\title{
A scaling limit for the length of the longest cycle in a sparse random digraph
}

\author{
Michael Anastos $^{1}$ | Alan Frieze
}

${ }^{1}$ Department of Mathematics and Computer Science, Freie Universität Berlin, Berlin, Germany ${ }^{2}$ Department of Mathematical Sciences, Carnegie Mellon University, Pittsburgh, Pennsylvania, USA

\section{Correspondence}

Michael Anastos, Department of Mathematics and Computer Science, Freie Universität Berlin,

Berlin, Germany.

Email: manastos@zedat.fu-berlin.de

\begin{abstract}
We discuss the length $\vec{L}_{c, n}$ of the longest directed cycle in the sparse random digraph $D_{n, p}, p=c / n, c$ constant. We show that for large $c$ there exists a function $\vec{f}(c)$ such that $\vec{L}_{c, n} / n \rightarrow \vec{f}(c)$ a.s. The function $\vec{f}(c)=1-\sum_{k=1}^{\infty} p_{k}(c) e^{-k c}$ where $p_{k}$ is a polynomial in $c$. We are only able to explicitly give the values $p_{1}, p_{2}$, although we could in principle compute any $p_{k}$.
\end{abstract}

\section{KEYWORDS}

longest cycle, random digraphs, scaling limit

\section{1 | INTRODUCTION}

In this article, we consider the length $\vec{L}_{c, n}$ of the longest cycle in the random digraph $D_{n, p}, p=c / n$ where we will assume that $c$ is a sufficiently large constant. Here $D_{n, p}$ is the random subgraph of the complete digraph $\vec{K}_{n}$ obtained by including each of the $n(n-1)$ edges independently with probability $p$. Most of the literature on long cycles has been concerned with the length $L_{c, n}$ of the longest cycle in the random graph $G_{n, p}$. It was shown by Frieze [9] that w.h.p. $L_{c, n} \geq\left(1-\left(c+1+\varepsilon_{c}\right) e^{-c}\right) n$ where $\varepsilon_{c} \rightarrow 0$ as $c \rightarrow \infty$. Using the elegant coupling argument of McDiarmid [14], we see that this implies that w.h.p. $\vec{L}_{c, n} \geq\left(1-\left(c+1+\varepsilon_{c}\right) e^{-c}\right) n$. This was improved by Krivelevich, Lubetzky, and Sudakov [13] who showed that w.h.p. $\vec{L}_{c, n} \geq\left(1-\left(2+\varepsilon_{c}\right) e^{-c}\right) n$. Recently, Anastos and Frieze [1] have shown that if $c$ is sufficiently large then w.h.p. $L_{c, n} \approx f(c) n$ as $n \rightarrow \infty$, for some function $f(c){ }^{1}$

In this article, we use the ideas of [1] and show that w.h.p. $\vec{L}_{c, n} \approx \vec{f}(c) n$ and compute the first few terms of $\vec{f}(c)=1-\sum_{k=1}^{\infty} p_{k}(c) e^{-k c}$ where $p_{k}(c)$ is a polynomial in $c$ for $k \geq 1$. That is, we prove a

${ }^{1}$ Here we say $A_{n} \approx B_{n}$ if $A_{n} / B_{n} \rightarrow 1$ as $n \rightarrow \infty$.

This is an open access article under the terms of the Creative Commons Attribution-NonCommercial-NoDerivs License, which permits use and distribution in any medium, provided the original work is properly cited, the use is non-commercial and no modifications or adaptations are made. () 2021 The Authors. Random Structures and Algorithms published by Wiley Periodicals LLC. 
scaling limit for $\vec{L}_{c, n}$. The important point here is that we establish high probability errors that tend to zero with $n$, regardless of $c$.

Let $K_{1}$ denote the giant strong component of $D_{n, p}$, as discovered by Karp [12]. We consider a process that builds a large Hamiltonian subgraph of $K_{1}$. Our aim is to construct (something close) to a copy of the random graph $D_{5-\text { in,5-out }}$ as a large subgraph of $K_{1}$. In the random graph $D_{k-\text { in }, k-o u t}$ each $v \in[n]$ independently chooses $k$ in-neighbors and $k$ out-neighbors to make a digraph with $\approx 2 k n$ random edges. It has been shown by Cooper and Frieze $[5,6]$ that $D_{k-i n, k-o u t}$ is Hamiltonian w.h.p. provided that $k \geq 2$. Taking $k=5$ as opposed to $k=2$ will greatly simplify the discussion. In order to do this, we will construct $D_{n, p}$ as the union of two independent copies $D_{\text {red }}, D_{\text {blue }}$ of $D_{n, q}$ where $1-p=(1-q)^{2}$ so that $q=\frac{c}{2 n}+O\left(n^{-2}\right)$. One copy will have red edges and the other copy will have blue edges. A red edge $(v, w)$ will be associated with the vertex $v$ and a blue edge $(v, w)$ will be associated with the vertex $w$. In this way, the vertex $v$ will be incident to a random number of red out-edges and to a random number of blue in-edges. These edge sets will be independent by construction. We say in the following that $w$ is a blue in-neighbor of $v$ if $(w, v)$ is an edge of $D_{b l u e}$ and that $w$ is a red out-neighbor of $v$ if $(v, w)$ is an edge of $D_{\text {red }}$.

We construct a sequence of sets $S_{0}=\emptyset, S_{1}, S_{2}, \ldots, S_{L} \subseteq K_{1}$ as follows: suppose now that we have constructed $S_{\ell}, \ell \geq 0$. We construct $S_{\ell+1}$ from $S_{\ell}$ via one of two cases:

\section{Construction of $S_{L}$}

Case a: If there is a vertex $v \in S_{\ell}$ that has at most four blue in-neighbors outside $S_{\ell}$ then we add the blue in-neighbors of $v$ outside $S_{\ell}$ to $S_{\ell}$ to make $S_{\ell+1}$. Similarly, if there is a vertex $v \in S_{\ell}$ that has at most four red out-neighbors outside $S_{\ell}$ then we add the red out-neighbors of $v$ outside $S_{\ell}$ to $S_{\ell}$ to make $S_{\ell+1}$.

Case b: If there is a vertex $v \in K_{1} \backslash S_{\ell}$ that has at most four blue in-neighbors in $K_{1} \backslash S_{\ell}$ then we add $v$ and the blue in-neighbors of $v$ to $S_{\ell}$ to make $S_{\ell+1}$. Similarly, if there is a vertex $v \in K_{1} \backslash S_{\ell}$ that has at most four red out-neighbors in $K_{1} \backslash S_{\ell}$ then we then we add $v$ and the red out-neighbors of $v$ to $S_{\ell}$ to make $S_{\ell+1}$.

$S_{L}$ is the set we end up with when there are no more vertices to add. We note that $S_{L}$ is well defined and does not depend on the order of adding vertices. Indeed, suppose we have two distinct outcomes $O_{1}=v_{1}, v_{2}, \ldots, v_{r}$ and $O_{2}=w_{1}, w_{2}, \ldots, w_{s}$. Assume without loss of generality that there exists $i$ which is the smallest index such that $w_{i} \notin O_{1}$. Then, $X=\left\{w_{1}, w_{2}, \ldots, w_{i-1}\right\} \subseteq O_{1}=\left\{v_{1}, v_{2}, \ldots, v_{r}\right\}$. If $w_{i}$ invoked Case a or Case $\mathrm{b}$ then $w_{i}$ has at most four blue in-neighbors or at most four red out-neighbors in $K_{1} \backslash X$ hence in $K_{1} \backslash O_{1} \subseteq K_{1} \backslash X$. This contradicts the fact that $w_{i} \notin O_{1}$. Otherwise $w_{i}$ was added to $X$ because there exists a vertex $u \in X$ such that $w_{i}$ is a blue in-neighbor (or a red out-neighbor, respectively) of $u$ and $u$ has at most four blue in-neighbors (red out-neighbors resp.) in $K_{1} \backslash X$. Thus $u \in O_{1}$ has at most four blue in-neighbors (red out-neighbors resp.) in $K_{1} \backslash X \subseteq K_{1} \backslash X$. Once again, this contradicts the fact that $w_{i} \notin O_{1}$.

We will argue below in Section 1.1 that w.h.p. the graph $\Gamma_{L}$ underlying the digraph $D_{L}$ induced by $S_{L}$ is a forest plus a few small components (the graph underlying a digraph is obtained by ignoring orientation). Each tree in $\Gamma_{L}$ will w.h.p. have at most $\log n$ vertices and w.h.p. $\Gamma_{L}$ will have $o(n)$ vertices lying on non-tree components. From now on, when we refer to trees, they are either trees of $\Gamma_{L}$ or digraphs whose underlying graphs are trees of $\Gamma_{L}$.

Notation 1. Let $\overrightarrow{\mathcal{T}}$ denote the set of trees in $\Gamma_{L}$. Each tree $T$ of $\Gamma_{L}$ will appear as a digraph $\vec{T}$ in $D_{L}$ when we take account of orientation. For $\vec{T} \in \overrightarrow{\mathcal{T}}$, let $\overrightarrow{\mathcal{P}}_{T}$ be the set of vertex disjoint packings of properly oriented paths in $\vec{T}$ where we allow only paths whose start vertex has blue in-neighbors in $K_{1} \backslash V(\vec{T})$ and whose end vertex has red out-neighbors in $K_{1} \backslash V(\vec{T})$. Here we allow paths of length 0 , so that a single vertex with neighbors in $K_{1} \backslash V(\vec{T})$ counts as a path. For $P \in \overrightarrow{\mathcal{P}}_{T}$, let $n(\vec{T}, P)$ be the number 
of vertices in $\vec{T}$ that are not covered by $P$. Let $\phi(\vec{T})=\min _{P \in \overrightarrow{\mathcal{P}}_{T}} n(\vec{T}, P)$ and $\overrightarrow{\mathcal{Q}}(\vec{T}) \in \overrightarrow{\mathcal{P}}$ denote a set of paths that leaves $\phi(\vec{T})$ vertices of $\vec{T}$ uncovered, that is, satisfies $n(\vec{T}, \overrightarrow{\mathcal{Q}}(\vec{T}))=\phi(\vec{T})$.

We will prove

Theorem 1.1. Let $p=c / n$ where $c>1$ is a sufficiently large constant. Then w.h.p.

$$
\vec{L}_{c, n} \approx\left|V\left(K_{1}\right)\right|-\sum_{\vec{T} \in \overrightarrow{\mathcal{T}}} \phi(\vec{T}) .
$$

The RHS of (1), modulo the $o(n)$ vertices that are spanned by non-tree components in $\Gamma_{L}$, is clearly an upper bound on the largest directed cycle in $K_{1}$. Any cycle must omit at least $\phi(\vec{T})$ vertices from each $\vec{T} \in \overrightarrow{\mathcal{T}}$. On the other hand, as we show below, w.h.p. there is cycle $H$ that spans $V^{*}=\left(K_{1} \backslash S_{L}\right) \cup$ $\bigcup_{T \in \mathcal{T}} V(\mathcal{Q}(T))$. The length of $H$ is equal to the RHS of (1).

The size of $K_{1}$ is well known. Let $x$ be the unique solution of $x e^{-x}=c e^{-c}$ in $(0,1)$. Then w.h.p. (see, e.g., [10, Theorem 13.2]),

$$
\left|K_{1}\right| \approx\left(1-\frac{x}{c}\right)^{2} n
$$

Equation (4.5) of Erdős and Rényi [8] tells us that

$$
x=\sum_{k=1}^{\infty} \frac{k^{k-1}}{k !}\left(c e^{-c}\right)^{k}=c e^{-c}+c^{2} e^{-2 c}+O\left(c^{3} e^{-3 c}\right) .
$$

We will argue below that w.h.p., as $c$ grows, that

$$
\sum_{\vec{T} \in \overrightarrow{\mathcal{T}}} \phi(\vec{T})=\left(c^{2} e^{-2 c}+O\left(c^{3} e^{-3 c}\right)\right) n .
$$

The term $c^{2} e^{-2 c} n$ arises from vertices of out-degree one sharing a common out-neighbor or vertices of in-degree one sharing a common in-neighbor.

We therefore have the following improvement to the estimate in [13].

Corollary 1.2. W.h.p., as c grows,

$$
\vec{L}_{c, n} \approx\left(1-2 e^{-c}-\left(c^{2}+2 c-1\right) e^{-2 c}-O\left(c^{3} e^{-3 c}\right)\right) n .
$$

Note the term $2 e^{-c}-e^{-2 c}$ accounts for vertices of in- or out-degree 0 . In principle, we can compute more terms than what is given in (5). We claim next that there exists some function $\vec{f}(c)$ such that the sum in (1) is concentrated around $\vec{f}(c) n$. In other words, the sum in (1) has the form $\approx \vec{f}(c) n$ w.h.p.

\section{Theorem 1.3.}

(a) There exists a function $\vec{f}(c)$ such that for any fixed $\epsilon>0$, there exists $n_{\varepsilon}$ such that for $n \geq n_{\varepsilon}$,

$$
\left|\frac{\mathbb{E}\left[\vec{L}_{c, n}\right]}{n}-\vec{f}(c)\right| \leq \epsilon .
$$


(b)

$$
\frac{\vec{L}_{c, n}}{n} \rightarrow \vec{f}(c) \text { a.s. }
$$

We will show that taking $c \geq 200$ in Theorems 1.1 and 1.3 suffices.

We will prove Theorem 1.3 in Section 3. We are grateful to a reviewer for pointing out that $\vec{L}_{c, n} / n \rightarrow$ $f(c)$ in $L^{r}, r \geq 1$ because $\vec{L}_{c, n} / n$ is an a.s. bounded random variable.

\section{1 | Structure of $D_{L}$}

We first bound the size of $S_{L}$. We need the following lemma on the density of small sets.

Lemma 1.4. W.h.p., every set $S \subseteq[n]$ of size at most $n_{0}=n / 100 c^{3}$ contains less than $3|S| / 2$ edges in $D_{n, p}$.

Proof. The expected number of sets invalidating the claim can be bounded by

$$
\begin{aligned}
\sum_{s=3}^{n_{0}}\left(\begin{array}{c}
n \\
s
\end{array}\right)\left(\begin{array}{c}
s(s-1) \\
3 s / 2
\end{array}\right)\left(\frac{c}{n}\right)^{3 s / 2} & \leq \sum_{s=3}^{n_{0}}\left(\frac{n e}{s} \cdot\left(\frac{2 s e}{3}\right)^{3 / 2} \cdot\left(\frac{c}{n}\right)^{3 / 2}\right)^{s} \\
& =\sum_{s=3}^{n_{0}}\left(\frac{e^{5 / 2}(2 c)^{3 / 2} s^{1 / 2}}{3^{3 / 2} n^{1 / 2}}\right)^{s}=o(1) .
\end{aligned}
$$

Now consider the construction of $S_{L}$. Let $A \subseteq K_{1}$ be the set of the vertices with blue in-degree less than $D=30$ or red out-degree less than $D$ in $K_{1}$. Let $S_{0}^{\prime}=\left(A \cup N_{b}(A) \cup N_{r}(A)\right) \cap S_{L} \subseteq S_{L}$, where $N_{b}(A)$ is the set of blue in-neighbors of vertices in $A$ and $N_{r}(A)$ is the set of red out-neighbors of vertices in $A$. If we start with $S_{0}=S_{0}^{\prime}$ and run the process for constructing $\Gamma_{L}$ then we will produce the same $S_{L}$ as if we had started with $S_{0}=\emptyset$. This is because, as we have shown, the order of adding vertices does not matter. Now w.h.p. there are at most $n_{D}=\frac{2 c^{D} e^{-c}}{D !} n$ vertices of blue in-degree at most $D$ or red out-degree at most $D$ (see, e.g., Theorem 3.3 of [10] that deals with the same question as it relates to degrees in $G_{n, p}$ ).

Now suppose that the process runs for another $k$ rounds. Then $S_{k}$ contains at least $k D$ edges and at most $D n_{D}+5 k$ vertices. This is because round $k$ adds at most five new vertices to $S_{k}$ and the $k$ vertices that take the role of $v$ have either (i) blue in-degree at least $D$ with all blue in-neighbors in $S_{k}$ or (ii) red out-degree at least $D$ with all red out-neighbors in $S_{k}$. If $k$ reaches $2 n_{D}$ then

$$
\frac{e\left(S_{k}\right)}{\left|S_{k}\right|} \geq \frac{2 D n_{D}}{(D+10) n_{D}}=\frac{3}{2} .
$$

So, by Lemma 1.4, we can assert that w.h.p. the process runs for less than $2 n_{D}$ rounds and,

$$
\left|V\left(\Gamma_{L}\right)\right| \leq(D+10) n_{D}=(D+10) \frac{2 c^{D} e^{-c}}{D !} n \leq 2(D+10)\left(\frac{e c}{D}\right)^{D} n e^{-c} \leq n e^{-c / 2} .
$$

The last inequality holds for $c \geq 200$ and $D=30$.

We note the following properties of $S_{L}$. Let

$$
\begin{aligned}
& V_{1}=K_{1} \backslash S_{L} \text { and } V_{2}=\left\{v \in S_{L}: v\right. \text { has at least one blue in-neighbor and at least one red } \\
& \text { out-neighbor in } \left.V_{1}\right\} \text {. }
\end{aligned}
$$


Then,

G1 Each vertex $v \in S_{L} \backslash V_{2}$ has no blue in-neighbors or no red out-neighbors in $V_{1}$.

G2 Each $v \in V_{1} \cup V_{2}$ has at least five blue in-neighbors and five red out-neighbors in $V_{1}$.

Now consider a component $K$ of $\Gamma_{L}$. Let $C_{0}=C_{0}(K)=\left\{v_{1}, v_{2}, \ldots, v_{L}\right\}$ denote the set of vertices in $K$ that are $v$ in some step in the construction of $D_{L}$, indexed by the round in which they are added. Since a vertex may invoke some step in the construction of $D_{L}$ at most twice we have,

$$
\left|C_{0}(K)\right| \geq L / 2
$$

At the same time, at each step the set $\left|K \backslash C_{0}(K)\right|$ may grow by at most 4 and so

$$
\left|K \backslash C_{0}(K)\right| \leq 4 L \leq 8\left|C_{0}(K)\right|
$$

Hence

$$
\left|C_{0}(K)\right| \geq \frac{|K|}{9}
$$

We next show that w.h.p., only a small component $K$ can satisfy (10). $K$ will have at least $|K| / 9$ vertices for which either there are no blue in-neighbors outside $K$ or no red out-neighbors outside of $K$. It will also contain a spanning tree in the graph underlying $D_{n, p}$. So, the expected number of components of size $k \leq n e^{-c / 2}$ that satisfy this condition is at most

$$
\begin{aligned}
\left(\begin{array}{l}
n \\
k
\end{array}\right) k^{k-2}\left(\frac{c}{n}\right)^{k-1}\left(\begin{array}{c}
k \\
k / 9
\end{array}\right) \times\left(2\left(1-\frac{c}{2 n}\right)^{(n-k)}\right)^{k / 9} & \leq\left(\frac{n e}{k}\right)^{k} k^{k-2}\left(\frac{c}{n}\right)^{k-1} 2^{10 k / 9} e^{-c k / 20} \\
& \leq \frac{n}{c k^{2}}\left(2^{10 / 9} c e^{1-c / 20}\right)^{k}=o\left(n^{-2}\right)
\end{aligned}
$$

if $c \geq 200$ and $k \geq \log n$.

So, we can assume that all components are of size at $\operatorname{most} \log n$. Then the expected number of vertices on components that are not trees is bounded by

$$
\begin{aligned}
\sum_{k=2}^{\log n}\left(\begin{array}{l}
n \\
k
\end{array}\right) k^{k+1}\left(\frac{c}{n}\right)^{k}\left(\begin{array}{c}
k \\
k / 9
\end{array}\right) \times\left(2\left(1-\frac{c}{2 n}\right)^{(n-k)}\right)^{k / 9} & \leq \sum_{k=2}^{\log n}\left(\frac{n e}{k}\right)^{k} k^{k+1}\left(\frac{c}{n}\right)^{k} 2^{10 k / 9} e^{-c k / 20} \\
& \leq \sum_{k=2}^{\log n} k\left(2^{10 / 9} c e^{1-c / 20}\right)^{k}=O(1)
\end{aligned}
$$

The Markov inequality implies that w.h.p. such components span at most $\log n=o(n)$ vertices.

\section{2 | PROOF OF THEOREM 1.1}

For $\vec{T} \in \overrightarrow{\mathcal{T}}$, let $\vec{X}_{T}$ be the set obtained by contracting each path $\vec{P}$ of $\overrightarrow{\mathcal{Q}}(\vec{T})$ to a vertex $v_{\vec{P}}$ with blue in-neighbors in $V_{1}$ equal to the blue in-neighbors in $V_{1}$ of the start vertex of $\vec{P}$ and red out-neighbors in $V_{1}$ equal to the red out-neighbors in $V_{1}$ of the end vertex of $\vec{P}$. Note that the colors of the internal edges of a path $\vec{P}$ do not play a role here. Let $\vec{X}^{*}=\bigcup_{\vec{T} \in \overrightarrow{\mathcal{T}}} \vec{X}_{T}$. By construction, the digraph induced by $V_{1}$ contains a copy of $D_{5-\text { in }, 5-\text { out }}$ with $N=\left|V_{1}\right|$ vertices. Indeed, the blue edges contributing the 5-in edges 
and the red edges contributing the 5-out edges. For each $v \in V_{1}$, the blue in-neighbors form a random set of size at least five, independent of the other vertices in $V_{1}$. Similarly for the red out-neighbors.

We let $D^{*}$ be the digraph with vertex set $V_{1}^{*}=V_{1} \cup \vec{X}^{*}$ and a copy of $D_{5-\text { in,5-out }}$ on $V_{1}$ and for each $x \in \vec{X}^{*}$ five red edges joining $x$ to $V_{1}$ and five blue edges from $V_{1}$ to $x$.

Our next task is to prove that the random digraph $D^{*}$ defined in the previous section contains a Hamilton cycle. Let $H$ denote such a cycle through $V_{1}^{*}$. We obtain a Hamilton cycle of $V^{*}$ (defined following Theorem 1.1) by uncontracting each path $\vec{P}$ of $\vec{Q}(\vec{T})$. This will complete the proof of Theorem 1.1. Our proof of the existence of $H$ will be very similar to the proof in Cooper and Frieze [7]. It does not really offer any new technical insights and so we have placed the proof into Appendix A.

\section{3 | PROOF OF THEOREM 1.3}

For $\vec{T} \in \overrightarrow{\mathcal{T}}$, we let $v_{0}(\vec{T})$ denote the set of vertices in $\vec{T}$ that do not have neighbors outside $\vec{T}$. For $v \in K_{1}$, we let $\phi(v)=\phi(\vec{T}) /\left|v_{0}(\vec{T})\right|$ if $v \in v_{0}(T)$ for some $\vec{T} \in \overrightarrow{\mathcal{T}}$ and $\phi(v)=0$ otherwise. Thus

$$
\sum_{T \in \overrightarrow{\mathcal{T}}} \phi(\vec{T})=\sum_{v \in K_{1}} \phi(v)
$$

Hence (1) can be rewritten as,

$$
\vec{L}_{c, n} \approx\left|K_{1}\right|-\sum_{v \in K_{1}} \phi(v)
$$

Let $k_{1}=k_{1}(\epsilon, c)$ be the smallest positive integer such that

$$
\sum_{k=k_{1}-1}^{\infty}\left(e^{9} 2^{11} c e^{-c / 5}\right)^{k}<\frac{\epsilon}{3}
$$

Note that for $\varepsilon \leq 1 / 2$ and $c \geq 200$, we have

$$
k_{1} \leq \frac{30}{c} \log \frac{1}{\varepsilon}
$$

as

$$
\sum_{k=k_{1}-1}^{\infty}\left(e^{9} 2^{11} c e^{-c / 5}\right)^{k} \leq 2\left(\left(e^{9} 2^{11} c\right)^{5 / c} e^{-1}\right)^{-6 \log \varepsilon} \leq 2\left(\left(e^{9} 2^{11} 200\right)^{5 / 200} e^{-1}\right)^{-6 \log \varepsilon}<\frac{\epsilon}{3} .
$$

To begin let $\vec{K}_{5,5}$ denote the complete bipartite digraph with ten vertices, five in each part of the partition. The arcs inside $\vec{K}_{5,5}$ are consider to have both colors, red and blue. For $v \in K_{1}$, let $D_{v}$ be the digraph consisting of the vertices of $D=D_{n, p}=D_{\text {blue }} \cup D_{\text {red }}$ that are within distance $k_{1}$ from $v$, where for every vertex $u$ in the $k_{1}$ neighborhood of $v$ we introduce a new copy of $\vec{K}_{5,5}$ and join $u$ to each vertex of the same one part of the bipartition of its $\vec{K}_{5,5}$ by a blue in-arc and a red out-arc from $u$. Distance here is graph distance in the undirected graph underlying $D$. We consider the algorithm for the construction of $\Gamma_{L}$ on $G_{v}$, the graph underlying $D_{v}$. Let $K_{1, v}, \Gamma_{L, v}, V_{1, v}, S_{L, v}, v_{0, v}(\vec{T})$ be the corresponding sets/quantities. 
For a tree $\vec{T} \in S_{L, v}$, let $\vec{f}(\vec{T})$ be equal to $|\vec{T}|$ minus the maximum number of vertices that can be covered by a set of vertex disjoint paths with endpoints in $V_{2, v}$ (we allow paths of length 0 ). For $v \in K_{1}$, if $v$ belongs to some tree $\vec{T} \in S_{L, v}$ set $\vec{f}(v)=\vec{f}(\vec{T}) / v_{0, v}(\vec{T})$, otherwise set $\vec{f}(v)=0$.

For $v \in K_{1}$, let $t(v)=1$ if $v \in V_{1}$ or if $v \in S_{L}$ and in $\Gamma_{L}, v$ lies in a component with at most $k_{1}-2$ vertices in $\Gamma_{L}$. Set $t(v)=0$ otherwise. Observe that if $t(v)=1$ then $\phi(v)=\vec{f}(v)$. Otherwise $|\phi(v)-\vec{f}(v)| \leq 1$.

By repeating the arguments used to prove (11) and (10), it follows that if $t(v)=0$ then $v$ lies on a subgraph spanned by some set of vertices $K$ of size at most $\log n$. In addition at least $(|K|-1) / 9$ vertices in $K \backslash\{v\}$ either do not have blue in-neighbors or red out-neighbors outside $K$. Thus the expected number of vertices $v$ satisfying $t(v)=0$ is bounded by

$$
\begin{aligned}
& \sum_{k=k_{1}-1}^{\log n} \sum_{j=k}^{9 k}\left(\begin{array}{l}
n \\
j
\end{array}\right)\left(\begin{array}{l}
j \\
k
\end{array}\right) j^{j-2}(2 p)^{j-1} \times\left(2\left(1-\frac{p}{2}\right)^{(n-j)}\right)^{k} \\
& \leq 2 n \sum_{k=k_{1}-1}^{\log ^{2} n} 9 k\left(\frac{e}{9 k}\right)^{9 k} 2^{9 k}(9 k)^{9 k-2}(2 c)^{k-1} 2^{k} e^{-c k / 5} \\
& \leq 2 n \sum_{k=k_{1}-1}^{\infty}\left(e^{9} 2^{11} c e^{-c / 5}\right)^{k}<\frac{\epsilon n}{3}
\end{aligned}
$$

A vertex $v \in[n]$ is good if the $i$ th level of its breadth first search (BFS) neighborhood has size at most $3(2 c)^{i} k_{1} / \epsilon$ for every $i \leq k_{1}$ and it is bad otherwise. Here the BFS is done on the graph underlying $D$. Because the expected size of the $i$ th neighborhood is $\approx(2 c)^{i}$ we have by the Markov inequality that $v$ is bad with probability at most $(1+o(1)) \varepsilon / 3 \leq \varepsilon / 2$ and so the expected number of bad vertices is bounded by $\varepsilon n / 2$. Thus

$$
\begin{aligned}
\mathbb{E}\left(\left|\sum_{v \in V} \phi(v)-\sum_{v \text { is good }} \vec{f}(v)\right|\right) & \leq \mathbb{E}\left(\left|\sum_{v \in V} \phi(v)-\sum_{v \in V} \vec{f}(v)\right|\right)+\mathbb{E}\left(\left|\sum_{v \text { is bad }} \vec{f}(v)\right|\right) \\
& \leq \mathbb{E}\left(\left|\sum_{v: t(v)=0}\right| \phi(v)-\vec{f}(v) \mid\right)+\mathbb{E}\left(\sum_{v \text { is bad }} 1\right) \\
& \leq \mathbb{E}\left(\sum_{v: t(v)=0} 1\right)+\frac{\epsilon n}{2} \\
& \leq \frac{\epsilon n}{3}+\frac{\epsilon n}{2}<\epsilon n .
\end{aligned}
$$

Let $\mathcal{H}_{\varepsilon}$ be the set of BFS neighborhoods that are good, that is, whose $i$ th levels are of size at most $3(2 c)^{i} k_{1} / \epsilon$ for every $i \leq k_{1}$. Every element of $\mathcal{H}_{\varepsilon}$ corresponds to a pair $\left(H, o_{H}\right)$ where $H$ is a digraph and $o$ is a distinguished vertex of $H$, that is considered to be the root. Also for $v \in K_{1}$ let $D\left(N_{k_{1}}(v)\right)$ be the subdigraph induced by the $k_{1}$ th neighborhood of $v$. For $\left(H, o_{H}\right) \in \mathcal{H}_{\varepsilon}$, let $\operatorname{int}(H)$ be the set of vertices incident to the first $k_{1}-1$ neighborhoods of $o_{H}$ and let $A u t\left(H, o_{H}\right)$ be the number of automorphisms of $H$ that fix $o_{H}$. Note that each good vertex $v$ is associated with a pair $\left(H, o_{H}\right) \in \mathcal{H}_{\varepsilon}$ from which we can compute $\vec{f}(v)$, since $\vec{f}(v)=\vec{f}\left(o_{H}\right)$. Thus, if now

$$
M=\left|E\left(K_{1}\right)\right|, N=\left|K_{1}\right|
$$




$$
\begin{aligned}
\mathbb{E}\left(\sum_{v \text { is good }} \vec{f}(v) \mid M, N\right) & =\sum_{v} \sum_{k \geq 1} \sum_{\begin{array}{c}
\left(H, o_{H}\right) \in \mathcal{H}_{\varepsilon} \\
\left(D\left(N_{k_{1}}(v), v\right)=\left(H, o_{H}\right)\right. \\
|V(H)|=k
\end{array}} \rho_{H, o_{H}} \vec{f}\left(o_{H}\right) \\
& =o(n)+\sum_{v} \sum_{k \geq 1} \sum_{\begin{array}{c}
\left(H, o_{H}\right) \in \mathcal{H}_{\varepsilon} \\
\text { is a tree } \\
\left(D\left(N_{k_{1}}(v)\right), v\right)=\left(H, o_{H}\right) \\
|V(H)|=k
\end{array}} \rho_{H, o_{H}} \vec{f}\left(o_{H}\right),
\end{aligned}
$$

where $\rho_{H, o_{H}}$ is the probability $\left(D\left(N_{k_{1}}(v)\right), v\right)=\left(H, o_{H}\right)$ in $K_{1}$. We show in Section 3.1 that

$$
\rho_{H, o_{H}} \approx \frac{1}{\operatorname{Aut}\left(H, o_{H}\right)}\left(\frac{N}{M}\right)^{k-1} \lambda^{2 k-2} \frac{e^{2 k \lambda}}{f_{1}(\lambda)^{2 k}},
$$

where $f_{1}$ is defined in (18) and $\lambda$ satisfies (19).

Finally observe that with the exception of the $o(n)$ term, all the terms in (14) are independent of $n$. We let

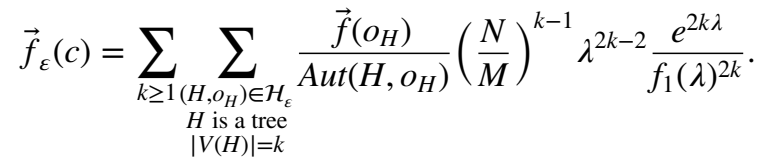

Then for a fixed $c$, we see that $\vec{f}_{\varepsilon}(c)$ is monotone increasing as $\varepsilon \rightarrow 0$. This is simply because $\mathcal{H}_{\varepsilon}$ grows. Furthermore, $\vec{f}_{\varepsilon}(c) \leq 1$ and so the limit $\vec{f}(c)=\lim _{\varepsilon \rightarrow 0} f_{\varepsilon}(c)$ exists. Let $S_{\varepsilon, n}$ be the number of vertices in $D_{n, p}$ (i) whose first $k_{1}$ neighborhoods are good and so total at most $4(2 c)^{k_{1}} k_{1} / \varepsilon-1$ vertices, and (ii) span a cycle in the underlying graph. The $o(n)$ term in (14) is bounded by $S_{\varepsilon, n}$. Hence, with $s=4(2 c)^{k_{1}} k_{1} / \varepsilon$, the $o(n)$ term is bounded by

$$
\sum_{i=1}^{s} i\left(\begin{array}{c}
n \\
i
\end{array}\right) i^{i-2}\left(\begin{array}{c}
i \\
2
\end{array}\right)(2 p)^{i} \leq \sum_{i=1}^{s} i\left(\frac{e n}{i}\right)^{i} i^{i}(2 p)^{i} \leq 2 s(2 e c)^{s} \leq \log \frac{1}{\varepsilon} \times e^{\log \frac{1}{\varepsilon}} \leq \frac{1}{\varepsilon^{2}},
$$

which depends only on $\varepsilon$.

This verifies part (a) of Theorem 1.3. For part (b), we prove, (see (30)),

\section{Lemma 3.1.}

$$
\mathbb{P}\left(\left|\vec{L}_{c, n}-\mathbb{E}\left(\vec{L}_{c, n}\right)\right| \geq \varepsilon n+n^{3 / 4}\right)=O\left(n^{-2}\right)
$$

Proof. To prove this, we show that if $v(H)$ is the number of copies of $H$ in $K_{1}$ then $H \in \mathcal{H}_{\varepsilon}$ implies that

$$
\mathbb{P}\left(|v(H)-\mathbb{E}(v(H))| \geq n^{3 / 5}\right)=O\left(n^{-3}\right) .
$$

The inequality follows from a version of Azuma's inequality (see (30)), and the lemma follows from taking a union bound over

$$
\begin{aligned}
\exp \left\{O\left(\frac{c^{k_{1}(\epsilon, c)} k_{1}(\epsilon, c)}{\epsilon}\right)\right\} & =\exp \left\{O\left(\frac{c^{\frac{30}{c} \log \frac{1}{\varepsilon} \frac{30}{c} \log \frac{1}{\varepsilon}}}{\varepsilon}\right)\right\} \\
& =\exp \left\{O\left(\frac{(1 / \varepsilon)^{\frac{30}{c} \log c} \log \frac{1}{\varepsilon}}{\varepsilon}\right)\right\}=\exp \left\{O\left((1 / \varepsilon)^{3}\right)\right\}
\end{aligned}
$$


graphs $H$. Note also that the $o(n)$ term in (14) is bounded by $S_{\varepsilon, n}$ and the probability that this exceeds $n^{1 / 2}$ is certainly at most the RHS of (17). We will give details of our use of the Azuma inequality in Section 3.1.

Part (b) of Theorem 1.3 follows by letting $\varepsilon \rightarrow 0$ and from the Borel-Cantelli lemma.

\subsection{A model of $K_{1}$}

$K_{1}$ induces a random digraph with minimum in-degree and out-degree at least one. $K_{1}$ is distributed as a random strongly connected digraph with $N$ vertices and $M$ edges. This follows from the fact that each such digraph has the same number of extensions to a digraph with $n$ vertices and $m$ edges where $K_{1}$ is the unique giant strongly connected component. Most vertices of $K_{1}$ will have in-degree and out-degree close to $c$, since $c$ is large. It follows from Theorem 3 of Cooper and Frieze [7] that a random digraph with this degree sequence has a probability of being strongly connected that is asymptotic to $e^{-\beta}$ where $\beta=\beta(c) \rightarrow 0$ as $c \rightarrow \infty$. It follows from this that we can model the digraph induced by $K_{1}$ as a random digraph with $N$ vertices and $M$ edges. The probability of any event will be inflated by at most $(1+o(1)) e^{\beta}$ by conditioning on strong connectivity. We denote this model by $D_{N, M}^{ \pm 1}$.

\subsection{1 | Random sequence model}

This is essentially a repeat of Section 3.1.1 of [1]. The differences are minor, but we feel we need to include the argument. We must now take some time to explain the model we use for $D_{N, M}^{ \pm 1}$. We use a variation on the pseudo-graph model of Bollobás and Frieze [3] and Chvátal [4]. Given a sequence $\mathbf{x}=\left(x_{1}, x_{2}, \ldots, x_{2 M}\right) \in[n]^{2 M}$ of $2 M$ integers between 1 and $N$, we can define a (multi)-digraph $D_{\mathbf{x}}=$ $D_{\mathbf{x}}(N, M)$ with vertex set $[N]$ and edge set $\left\{\left(x_{2 i-1}, x_{2 i}\right): 1 \leq i \leq M\right\}$. The in-degree $d_{\mathbf{x},-}(v)$ of $v \in[N]$ and the out-degree $d_{\mathbf{x},+}(v)$ of $v \in[N]$ are given by

$$
d_{\mathbf{x},-}(v)=\left|\left\{j \in[M]: x_{2 j}=v\right\}\right| \text { and } d_{\mathbf{x},+}(v)=\left|\left\{j \in[M]: x_{2 j-1}=v\right\}\right| .
$$

If $\mathbf{x}$ is chosen randomly from $[N]^{2 M}$ then $D_{\mathbf{x}}$ is close in distribution to $D_{N, M}$. Indeed, conditional on being simple, $D_{\mathbf{x}}$ is distributed as $D_{N, M}$. To see this, note that if $D_{\mathbf{x}}$ is simple then it has vertex set [N] and $M$ edges. Also, there are $M$ ! distinct equally likely values of $\mathbf{x}$ which yield the same digraph.

Our situation is complicated by there being a lower bound of one on the minimum in-degree and out-degree. So we let

$$
[N]_{\delta \pm \geq 1}^{2 M}=\left\{\mathbf{x} \in[N]^{2 M}: d_{\mathbf{x}, \pm}(j) \geq 1 \text { for } j \in[N]\right\}
$$

Let $D_{\mathbf{x}}$ be the multi-graph $D_{\mathbf{x}}$ for $\mathbf{x}$ chosen uniformly from $[N]_{\delta \pm \geq 1}^{2 M}$. It is clear then that conditional on being simple, $D_{\mathbf{x}}$ has the same distribution as $D_{N, M}^{ \pm 1}$. It is important therefore to estimate the probability that this graph is simple. For this and other reasons, we need to have an understanding of the degree sequence $d_{\mathbf{x}}$ when $\mathbf{x}$ is drawn uniformly from $[N]_{\delta \pm \geq 1}^{2 M}$. Let

$$
f_{1}(\lambda)=e^{\lambda}-1
$$

Lemma 3.2. Let $\mathbf{x}$ be chosen randomly from $[N]_{\delta \pm \geq 1}^{2 M}$. Let $Y_{j}, Z_{j}, j=1,2, \ldots, N$ be independent copies of a truncated Poisson random variable $\mathcal{P}$, where

$$
\mathbb{P}(\mathcal{P}=t)=\frac{\lambda^{t}}{t ! f_{1}(\lambda)}, \quad t \geq 1 .
$$


Here $\lambda$ satisfies

$$
\frac{\lambda e^{\lambda}}{f_{1}(\lambda)}=\frac{M}{N}
$$

Then $\left\{d_{\mathbf{x},-}(j)\right\}_{j \in[N]}$ is distributed as $\left\{Y_{j}\right\}_{j \in[N]}$ conditional on $Y=\sum_{j \in[n]} Y_{j}=M$ and $\left\{d_{\mathbf{x},+}(j)\right\}_{j \in[N]}$ is distributed as $\left\{Z_{j}\right\}_{j \in[N]}$ conditional on $Z=\sum_{j \in[n]} Z_{j}=M$.

Proof. This can be derived as in Lemma 4 of [2].

We note that w.h.p.

$$
N \geq n\left(1-2 e^{-c / 2}\right) \text { and } M \in\left(1 \pm \varepsilon_{1}\right) c N
$$

where $\varepsilon_{1}=c^{-1 / 3}$. The bound on $N$ follows from (2) and (7) and the bound on $M$ follows from the fact that in $G_{n, p}$,

$$
\mathbb{P}\left(\exists S:|S|=N, e(S) \notin\left(1 \pm \varepsilon_{1}\right) N(N-1) p\right) \leq 2\left(\begin{array}{c}
n \\
N
\end{array}\right) \exp \left\{-\frac{\varepsilon_{1}^{2} N(N-1) p}{3}\right\}=o(1) .
$$

It follows from (19) and (20) and the fact that $e^{\lambda} / f_{1}(\lambda) \rightarrow 1$ as $c \rightarrow \infty$ that for large $c$,

$$
\lambda=c\left(1+O\left(e^{-c}\right)\right) \text {. }
$$

We note that the variance $\sigma^{2}$ of $\mathcal{P}$ is given by

$$
\sigma^{2}=\frac{\lambda(\lambda+1) e^{\lambda} f_{1}(\lambda)-\lambda^{2} e^{2 \lambda}}{f_{1}^{2}(\lambda)}
$$

Furthermore,

$$
\begin{gathered}
\mathbb{P}\left(\sum_{j=1}^{N} Y_{j}=M\right)=\frac{1}{\sigma \sqrt{2 \pi N}}\left(1+O\left(N^{-1} \sigma^{-2}\right)\right) \text { and } \\
\mathbb{P}\left(\sum_{j=2}^{N} Y_{j}=M-d\right)=\frac{1}{\sigma \sqrt{2 \pi N}}\left(1+O\left(\left(d^{2}+1\right) N^{-1} \sigma^{-2}\right)\right) .
\end{gathered}
$$

This is an example of a local central limit theorem. See, for example, (5) of [2]. It follows by repeated application of (22) and (23) that if $k=O(1)$ and $d_{1}^{2}+\ldots+d_{k}^{2}=o(N)$ then

$$
\mathbb{P}\left(Y_{i}=d_{i}, i=1,2, \ldots, k \mid \sum_{j=1}^{N} Y_{j}=M\right) \approx \prod_{i=1}^{k} \frac{\lambda^{d_{i}}}{d_{i} ! f_{1}(\lambda)}
$$

Let $v_{\mathbf{x},-}(s)$ denote the number of vertices of in-degree $s$ in $D_{\mathbf{x}}$ and let $v_{\mathbf{x},+}(s)$ denote the number of vertices of out-degree $s$ in $D_{\mathbf{x}}$.

Lemma 3.3. Suppose that $\log N=O\left((N \lambda)^{1 / 2}\right)$. Let $\mathbf{x}$ be chosen randomly from $[N]_{\delta \geq 2}^{2 M}$. Then as in equation (7) of [2], we have that with probability $1-o\left(N^{-10}\right)$,

$$
\left|v_{\mathbf{x}, \pm}(j)-\frac{N \lambda^{j}}{j ! f_{1}(\lambda)}\right| \leq\left(1+\left(\frac{N \lambda^{j}}{j ! f_{1}(\lambda)}\right)^{1 / 2}\right) \log ^{2} N, 1 \leq j \leq \log N
$$




$$
v_{\mathbf{x}}(j)=0, \quad j \geq \log N
$$

We can now show that $D_{\mathbf{x}}, \mathbf{x} \in[N]_{\delta \pm \geq 1}^{2 M}$ is a good model for $D_{N, M}^{ \pm 1}$. For this, we only need to show now that

$$
\mathbb{P}\left(D_{\mathbf{x}} \text { is simple }\right)=\Omega(1)
$$

Again, this follows as in [2].

Given a tree $H$ with $k$ vertices of in-degrees $y_{1}, y_{2}, \ldots, y_{k}$ and out-degrees $z_{1}, z_{2}, \ldots, z_{k}$ and a fixed vertex $v$ we see that if $\rho_{H}$ is the probability that $D\left(N_{k_{1}}(v)\right)=H$ in $D_{\mathbf{x}}$ then we have

$$
\begin{aligned}
\rho_{H} & \approx\left(\begin{array}{c}
N \\
k-1
\end{array}\right) \frac{(k-1) !}{A u t\left(H, o_{H}\right)} \sum_{D^{-}, D^{+}=k-1}^{\infty} \\
& \sum_{\substack{d_{1}^{-} \geq y_{1}, \ldots, d_{-}^{-} \geq y_{k} \\
d_{1}+\ldots+d_{k}=D^{-} \\
d_{1}^{+} \geq z_{1}, \ldots, d_{k}^{+} \geq z_{k} \\
d_{1}^{+}+\ldots+d_{k}^{+}=D^{+}}}^{k} \frac{\lambda^{d_{i}^{-}+d_{i}^{+}}}{d_{i}^{-} ! d_{i}^{+} ! f_{1}(\lambda)^{2}}\left(\begin{array}{c}
M \\
k-1
\end{array}\right)(k-1) ! \prod_{i=1}^{k} \frac{d_{i}^{-} ! d_{i}^{+} !}{\left(d_{i}^{-}-y_{i}\right) !\left(d_{i}^{+}-z_{i}\right) !} \frac{1}{M^{2 k-2}} \\
& \approx\left(\frac{N}{M}\right)^{k-1} \frac{\lambda^{2 k-2}}{\operatorname{Aut}\left(H, o_{H}\right) f_{1}(\lambda)^{2 k}} \sum_{d_{1}^{-}+\ldots+d_{k}^{-}=D^{-}} \prod_{i=1}^{k} \frac{\lambda^{d_{i}^{-}+d_{i}^{+}-y_{i}-z_{i}}}{\left(d_{i}^{-}-y_{i}\right) !\left(d_{1}^{+}-z_{i}\right) !} \\
& =\left(\frac{N}{M}\right)^{k-1} \frac{\lambda^{2 k-2}+\ldots+d_{k}^{+}=D^{+}}{\operatorname{Aut}\left(H, o_{H}\right) f_{1}(\lambda)^{2 k}}\left(\sum_{D=k-1}^{\infty} \frac{(k \lambda)^{D-(k-1)}}{(D-(k-1)) !}\right)^{2} \\
& \approx \frac{1}{\operatorname{Aut}\left(H, o_{H}\right)}\left(\frac{N}{M}\right)^{k-1} \lambda^{2 k-2} \frac{e^{2 k \lambda}}{f_{1}(\lambda)^{2 k}} .
\end{aligned}
$$

Explanation for (28): We use (24) to obtain the probability that the in-degrees and out-degrees of $[k]$ are $d_{1}^{-}, d_{1}^{+}, \ldots, d_{k}^{-}, d_{k}^{+}$. This accounts for the term $\prod_{i=1}^{k} \frac{\lambda^{d_{i}^{-}+d_{i}^{+}}}{d_{i}^{-} ! d_{i}^{+} ! f_{1}(\lambda)^{2}}$. Implicit here is that $d_{i}^{-}, d_{i}^{+}=$ $O(\log n)$, from (26). The contributions to the sum of $D^{-}, D^{+} \geq k \log n$ can therefore be shown to be negligible. We use the fact that $k$ is small to argue that w.h.p. $H$ is induced. We choose the vertices, other than $v$ in $\left(\begin{array}{c}N \\ k-1\end{array}\right)$ ways and then $\frac{(k-1) !}{\operatorname{Aut}\left(H, o_{H}\right)}$ counts the number of copies of $H$ in $K_{k}$. We then choose the place in the sequence to put these edges in $\left(\begin{array}{c}M \\ k-1\end{array}\right)(k-1)$ ! ways. Finally, note that the probability the $y_{i}$ occurrences of the $i$ th vertex are as claimed is asymptotically equal to $\frac{d_{i}^{-}\left(d_{i}^{-}-1\right) \ldots\left(d_{i}^{-}-y_{i}+1\right)}{M^{z_{i}}}$ and this explains the factor $\prod_{i=1}^{k} \frac{d_{i}^{-} ! d_{i}^{+} !}{\left(d_{i}^{-}-y_{i}\right) !\left(d_{i}^{+}-z_{i}\right) !} \frac{1}{M^{2 k-2}}$.

Explanation for (29): We use the identity

$$
\sum_{\substack{d_{1}, \ldots, d_{k} \\ d_{1}+\ldots+d_{k}=D}} \frac{D !}{d_{1} ! \ldots d_{k} !}=k^{D}
$$

It only remains to verify (17). It follows from the above that $\mathbb{E}(\nu(H) \mid M, N)=\Omega(N)$. We first condition on a degree sequence $\mathbf{x}$ satisfying (25). Then we condition on no element $\log n$ times or more in $\mathbf{x}$. 
The latter occurs with probability

$$
O\left(n^{1 / 2} e^{-\lambda} \frac{\lambda^{\log n}}{\log n !}\right)=O\left(n^{1 / 2} e^{-\lambda}\left(\frac{e \lambda}{\log n}\right)^{\log n}\right)=O\left(n^{-3}\right)
$$

Interchanging two elements in a permutation can only change $v(H)$ by $(\log n)^{k_{1}}=n^{o(1)}$. We can therefore apply Azuma's inequality to show that

$$
\mathbb{P}\left(|v(H)-\mathbb{E}(v(H))| \geq n^{3 / 5}\right)=O\left(e^{-\Omega\left(n^{1 / 5-o(1)}\right)}\right)+O\left(n^{-3}\right)=O\left(n^{-3}\right) .
$$

(Specifically, we can use Lemma 11 of Frieze and Pittel [11] or Section 3.2 of McDiarmid [15].) This verifies (17).

\section{ACKNOWLEDGMENTS}

Alan Frieze is supported in part by NSF Grant DMS1661063. Open access funding enabled and organized by Projekt DEAL.

\section{REFERENCES}

1. M. Anastos and A. M. Frieze, A scaling limit for the length of the longest cycle in a sparse random graph, J. Comb. Theory Ser. B 148 (2021), 184-208.

2. J. Aronson, A. M. Frieze, and B. G. Pittel, Maximum matchings in sparse random graphs: Karp-Sipser re-visited, Random Struct. Algorithms 12 (1998), 111-178.

3. B. Bollobás and A. M. Frieze, On matchings and Hamiltonian cycles in random graphs, Ann. Discrete Math. 28 (1985), 23-46.

4. V. Chvátal, Almost all graphs with 1.44n edges are 3-colourable, Random Struct. Algorithms 2 (1991), 11-28.

5. C. Cooper and A. M. Frieze, Hamilton cycles in a class of random directed graphs, J. Comb. Theory B 62 (1994), $151-163$.

6. C. Cooper and A. M. Frieze, Hamilton cycles in random graphs and directed graphs, Random Struct. Algorithms 16 (2000), 369-401.

7. C. Cooper and A. M. Frieze, The size of the largest strongly connected component of a random digraph with a given degree sequence, Comb. Probab. Comput. 13 (2004), 319-338.

8. P. Erdős and A. Rényi, On the evolution of random graphs, Publ. Math. Inst. Hungar. Acad. Sci. 5 (1960), 17-61.

9. A. M. Frieze, On large matchings and cycles in sparse random graphs, Discrete Math. 59 (1986), $243-256$.

10. A. M. Frieze and M. Karoński, Introduction to random graphs, Cambridge University Press, Cambridge, MA, 2015.

11. A. M. Frieze and B. Pittel, Perfect matchings in random graphs with prescribed minimal degree, in Trends in mathematics, Birkhauser Verlag, Basel, 2004, 95-132.

12. R. Karp, The transitive closure of a random digraph, Random Struct. Algorithms 1 (1990), 73-93.

13. M. Krivelevich, E. Lubetzky, and B. Sudakov, Longest cycles in sparse random digraphs, Random Struct. Algorithms 43 (2013), 1-15.

14. C. McDiarmid, Clutter percolation and random graphs, Math. Program. 13 (1980), 17-25.

15. C. McDiarmid, Concentration, in Probabilistic methods for algorithmic discrete mathematics, M. Habib, C. McDiarmid, J. Ramirez-Alfonsin, and B. Reed, Eds., Springer, Berlin, Germany, 1998, 1-46.

How to cite this article: M. Anastos, and A. Frieze, A scaling limit for the length of the longest cycle in a sparse random digraph, Random Struct. Algorithms. 60 (2022), 3-24. https://doi.org/10.1002/rsa.21030 
APPENDIX A: PROOF THAT $D^{*}$ IS HAMILTONIAN W.H.P.

The proof can be broken into three parts: suppose that $\left|V_{1}^{*}\right|=N=N_{1}+N_{2}$ where

$$
N_{1}=\left|V_{1}\right| \geq N\left(1-e^{-c / 2}\right) .
$$

(a) Find a collection $\Pi_{1}$ of $O(\log N)$ vertex disjoint directed cycles that cover $V_{1}^{*}$.

(b) Transform $\Pi_{1}$ into a collection $\Pi_{2}$ of vertex disjoint cycles such that each cycle is of length at least $N_{0}=\left\lceil\frac{200 N}{\log N}\right\rceil$.

(c) Break up $\Pi_{2}$ and reassemble it as a Hamilton cycle.

\section{A.1 Constructing $\Pi_{1}$}

Each vertex of $D^{*}$ is associated with five blue and five red edges. We randomly select three of each color and make them light and the rest heavy. We let $D_{3}$ be the digraph spanned by the light edges. We now consider the bipartite graph $H$ with bipartition made up of two copies $A, B$ of $V_{1}^{*}$ and an edge $\{v, w\}$ iff $(v, w)$ is a light edge. We show that w.h.p. $H$ contains a perfect matching. In the context of $D^{*}$, this gives us the collection of vertex disjoint directed cycles that cover $V_{1}^{*}$. We refer to this as a permutation digraph. We will argue that w.h.p. the number of cycles in the collection is $O(\log N)$. The probability that $H$ has no perfect matching can be bounded by

$$
\begin{aligned}
2 & \sum_{k=4}^{N / 2} \sum_{k_{1}=0}^{k} \sum_{k_{2}=0}^{k}\left(\begin{array}{c}
N_{1} \\
k_{1}
\end{array}\right)\left(\begin{array}{c}
N_{1} \\
k_{2}
\end{array}\right)\left(\begin{array}{c}
N_{2} \\
k-k_{1}
\end{array}\right)\left(\begin{array}{c}
N_{2} \\
k-k_{2}
\end{array}\right)\left(\frac{k_{2}}{N_{1}}\right)^{3 k}\left(1-\frac{k_{1}}{N_{1}}\right)^{3(N-k)} \\
& \leq 2 \sum_{k=4}^{N / 2 e^{2}} \sum_{k_{1}=0}^{k} \sum_{k_{2}=0}^{k}\left(\begin{array}{c}
N \\
k
\end{array}\right)\left(\begin{array}{c}
N \\
k
\end{array}\right)\left(\frac{k}{N_{1}}\right)^{3 k}+2 \sum_{N / 2 e^{2}}^{N / 2} \sum_{k_{1}=0}^{k} \sum_{k_{2}=0}^{k}\left(\begin{array}{c}
N \\
k
\end{array}\right)\left(\begin{array}{c}
N \\
k
\end{array}\right)\left(\frac{k}{N_{1}}\right)^{3 k} e^{-1.5 k \times k_{1} / k} \\
& \leq 2 \sum_{k=4}^{N / 2 e^{2}} k^{2}\left(\frac{e N}{k}\right)^{2 k}\left(\frac{k}{N_{1}}\right)^{3 k}+2 \sum_{N / 2 e^{2}}^{N / 2} k^{2}\left(\frac{e N}{k}\right)^{2 k}\left(\frac{k}{N_{1}}\right)^{3 k} e^{-1.5 k \times 0.9} \\
& \leq 2 \sum_{k=4}^{N / 2 e^{2}} k^{2}\left(\frac{k}{\left(1-e^{-c / 2}\right) N}\right)^{k}+2 \sum_{k=N / 2 e^{2}}^{N / 2} k^{2}\left(\frac{e^{0.65} k}{\left(1-e^{-c / 2}\right) N}\right)^{k}=o(1) .
\end{aligned}
$$

Explanation for (A1): We employ Hall's theorem. We choose a set $S \subseteq A$ of size $k \leq N / 2$ and a set $T \subseteq B$ also of size $k$. (No need to make $|T|=k-1$ here.) We let $k_{1}=\left|S \cap V_{1}\right|$ and $k_{2}=$ $\left|T \cap V_{1}\right|$. The number of ways of choosing these sets is given by the product of binomial coefficients. We then estimate the probability that $T \supseteq N(S)$. Each vertex in $S \cap A$ has probability at most $\left(\frac{k_{2}}{N_{1}}\right)^{3}$ of choosing all of its neighbors in $V_{1} \cap T$, explaining the factor $\left(\frac{k_{2}}{N_{1}}\right)^{3 k}$. Each vertex in $B \backslash T$ has probability $\left(1-\frac{k_{1}}{N_{1}}\right)^{3}$ of not choosing any neighbors in $V_{1} \cap S$, explaining the term $\left(1-\frac{k_{1}}{N_{1}}\right)^{3(N-k)}$. In the third line of the above calculations, we used the fact that if $k \geq N / 2 e^{2}$ then $k_{1} \geq k-e^{-c / 2} n \geq k-e^{-c / 2} N /$ $\left(1-e^{-c / 2}\right) \geq 0.9 k$.

This deals with $k \leq N / 2$ and if $k>N / 2$ then $B \backslash T$ and $A \backslash S$ can take the place of $S, T$, respectively.

We now consider the number of cycles in cycle cover induced by a matching in $H$. Suppose we write $M=\{(m(i), i): i \in B\}$ for some permutation $m$ of $A$. Further let $A=A_{1} \cup A_{X}$ where $A_{1}=\left\{a_{1}, a_{2}, \ldots, a_{N_{1}}\right\}$ corresponds to $V_{1}$ and $A_{X}$ corresponds to $\vec{X}^{*}$. We assume an analogous decomposition for $B$. Given a permutation $m$, we let $B_{X}(m)=\left\{b \in B: m(b) \in A_{X}\right\} \subseteq B_{1}$. The set inclusion 
follows from the fact that vertices in $A_{X}$ only have neighbors in $B_{1}$. Suppose now that we assume after relabeling that that $A, B$ are disjoint copies of $\left[N_{1}\right]$ and that $B_{X}(m), A_{X}$ are disjoint copies of $\left[N_{2}\right]$. Thus $m$ induces a permutation of $\left[N_{2}\right]$ and a permutation of $\left[N_{2}+1, N\right]$. We claim that conditional on this that $m$ induces uniform random permutations on these two sets. Suppose now that $m_{1}, m_{2}$ are two permutations that satisfy $m_{i}\left(\left[N_{2}\right]\right)=\left[N_{2}\right]$ for $i=1,2$. For a permutation $\pi$ of $A$ that satisfies $\left.\pi\left(\left[N_{2}\right]\right)\right)=\left[N_{2}\right]$ and graph $H$ we let $\pi(H)$ be obtained from $H$ by replacing edge $\{i, j\}$ by $\{\pi(i), j\}$. We note that $H$ and $\pi(H)$ have the same distribution. But then where $\pi(a)=m_{2}\left(m_{1}^{-1}(a)\right)$ for $a \in A$ we have

$$
\mathbb{P}\left(m(H)=m_{1}\right)=\mathbb{P}\left(m(\pi(H))=m_{2}\right)=\mathbb{P}\left(m(H)=m_{2}\right),
$$

justifying our uniformity claim.

Now a uniform random permutation on a set of size $M$ has $O(\log M)$ cycles w.h.p. It follows that w.h.p. the number of cycles induced by the matching constructed in $H$ has $O(\log N)$ cycles as claimed previously.

\section{A.2 $\mid$ Constructing $\Pi_{2}$}

We now show how to boost the minimum cycle size to at least $N_{0}$. We partition the cycles of the permutation digraph $\Pi_{1}$ into sets SMALL and LARGE, containing cycles $C$ of length $|C|<N_{0}$ and $|C| \geq N_{0}$, respectively. We define a near permutation digraph (NPD) to be a digraph obtained from a permutation digraph by removing one edge. Thus an NPD $\Gamma$ consists of a path $P(\Gamma)$ plus a permutation digraph $P D(\Gamma)$ which covers $[N] \backslash V(P(\Gamma))$.

We now give an informal description of a process which removes a small cycle $C$ from a current permutation digraph $\Pi$. We start by choosing an (arbitrary) edge $\left(v_{0}, u_{0}\right)$ of $C$ and delete it to obtain an NPD $\Gamma_{0}$ with $P_{0}=P\left(\Gamma_{0}\right) \in \mathcal{P}\left(u_{0}, v_{0}\right)$, where $\mathcal{P}(x, y)$ denotes the set of paths from $x$ to $y$ in $D$. The aim of the process is to produce a large set $S$ of NPD's such that for each $\Gamma \in S$, (i) $P(\Gamma)$ has a least $N_{0}$ edges and (ii) the small cycles of $P D(\Gamma)$ are a subset of the small cycles of $\Pi$. We will show that whp the endpoints of one of the $P(\Gamma)$ 's can be joined by an edge to create a permutation digraph with (at least) one less small cycle.

We have so far used six of the edges available at each vertex of $D^{*}$, namely those in $D_{3}$. We now let $D_{4}$ denote the 1-in, 1-out digraph associated with an unused fourth in- and out-edge associated with each vertex of $D^{*}$. Each vertex $v \in V^{*}$ will be associated with a random in-neighbor $i_{4}(v)$ and a random out-neighbor $\mathrm{Out}_{4}(v)$.

The basic step in an Out-Phase of this process is to take an NPD $\Gamma$ with $P(\Gamma) \in \mathcal{P}\left(u_{0}, v\right)$ and to examine the edges of $D_{4}$ leaving $v$, that is, edges going out from the end of the path. Let $w$ be the terminal vertex of such an edge and assume that $\Gamma$ contains an edge $(x, w)$. Then $\Gamma^{\prime}=\Gamma \cup\{(v, w)\} \backslash\{(x, w)\}$ is also an NPD. $\Gamma^{\prime}$ is acceptable if (i) $P\left(\Gamma^{\prime}\right)$ contains at least $N_{0}$ edges and (ii) any new cycle created (i.e., in $\Gamma^{\prime}$ and not $\Gamma$ ) also has at least $N_{0}$ edges.

If $\Gamma$ contains no edge $(x, w)$ then $w=u_{0}$. We accept the edge if $P$ has at least $N_{0}$ edges. This would (prematurely) end an iteration, by closing a cycle, although it is unlikely to occur.

We do not want to look at very many edges of $D_{4}$ in this construction and we build a tree $T_{0}$ of NPD's in a natural breadth-first fashion where each non-leaf vertex $\Gamma \in T_{0}$ gives rise to NPD children $\Gamma^{\prime}$ as described above. The construction of $T_{0}$ ends when we first have $v=\lceil\sqrt{N \log N}\rceil$ leaves. The construction of $T_{0}$ constitutes an Out-Phase of our procedure to eliminate small cycles. Having constructed $T_{0}$ we need to do a further In-Phase, which is similar to a set of Out-Phases.

Then w.h.p. we close at least one of the paths $P(\Gamma)$ to a cycle of length at least $N_{0}$. If $|C| \geq 4$ and this process fails then we try again with a different independent edge of $C$ in place of $\left(u_{0}, v_{0}\right)$. 
We now increase the formality of our description. We start Phase 2 with a permutation digraph $\Pi_{0}$ and a general iteration of Phase 2 starts with a permutation digraph $\Pi$ whose small cycles are a subset of those in $\Pi_{0}$. Iterations continue until there are no more small cycles. At the start of an iteration we choose some small cycle $C$ of $\Pi$. There then follows an Out-Phase in which we construct a tree $T_{0}=T_{0}(\Pi, C)$ of NPD's as follows: the root of $T_{0}$ is $\Gamma_{0}$ which is obtained by deleting an edge $\left(v_{0}, u_{0}\right)$ of $C$.

We grow $T_{0}$ to a depth at most $\lceil 1.5 \log n\rceil$. The set of nodes at depth $t$ is denoted by $S_{t}$.

Let $\Gamma \in S_{t}$ and $P=P(\Gamma) \in \mathcal{P}\left(u_{0}, v\right)$. A potential child $\Gamma^{\prime}$ of $\Gamma$, at depth $t+1$ is defined as follows.

Let $w$ be the terminal vertex of an edge directed from $v$ in $D_{4}$.

Case 1. $w$ is a vertex of a cycle $C^{\prime} \in P D(\Gamma)$ with edge $(x, w) \in C^{\prime}$. Let $\Gamma^{\prime}=\Gamma \cup\{(v, w)\} \backslash\{(x, w)\}$.

Case 2. $w$ is a vertex of $P(\Gamma)$. Either $w=u_{0}$, or $(x, w)$ is an edge of $P$. In the former case, $\Gamma \cup\{(v, w)\}$ is a permutation digraph $\Pi^{\prime}$ and in the latter case we let $\Gamma^{\prime}=\Gamma \cup\{(v, w)\} \backslash\{(x, w)\}$.

In fact, we only admit to $S_{t+1}$ those $\Gamma^{\prime}$ which satisfy the following conditions. We define a set $W$ of used vertices. Initially, all vertices are unused, that is, $W=\emptyset$. Whenever we examine an edge $(v, w)$, we add both $v$ and $w$ to $W$. So if $v \notin W$ then $\operatorname{out}_{4}(v)$ is still unconditioned and $\operatorname{in}_{4}(v)$ is a random member of a set $U \supseteq V^{*} \backslash W$. We do not allow $|W|$ to exceed $N^{3 / 4}$.

C(i) The new cycle formed (Case 2 only) must have at least $N_{0}$ vertices, and the path formed (both cases) must either be empty or have at least $N_{0}$ vertices. When the path formed is empty we close the iteration and if necessary start the next with $\Pi^{\prime}$.

C(ii) $x, w \notin W$.

An edge $(v, w)$ which satisfies the above conditions is described as acceptable.

We let $S_{t}$ be the set of endpoints of paths in $S_{t}$ that are not $u_{0}$. If some $N P D \in \mathcal{S}_{t}$ is the union of cycles then we are done with the given iteration. Thus we may assume otherwise and therefore $\left|S_{t}\right|=\left|S_{t}\right|$.

We also let $S_{t}^{1}=S_{t} \cap V_{1}$ and $S_{2}^{t}=S_{t} \backslash S_{1}^{t}$.

\section{Lemma A.1. Let $C \in S M A L L$. Then, where $v=\lceil\sqrt{N \log N}\rceil$,}

$$
\mathbb{P}\left(\exists t<\left\lceil\log _{1.9} v+1000 \log \log N\right\rceil \text { such that }\left|S_{t}\right| \in[v, 3 v]\right)=1-O\left((\log \log N)^{3} / \log N\right)
$$

Proof. We assume we stop an iteration, in mid-phase if necessary, when $\left|S_{t}\right| \in[v, 3 v]$. Let us consider a generic construction in the growth of $T_{0}$. Thus suppose we are extending from $\Gamma$ and $P(\Gamma) \in$ $\mathcal{P}\left(u_{0}, v\right)$.

We consider $S_{t+1}$ to be constructed in the following manner: we first examine $\operatorname{out}_{4}(v), v \in S_{t}$ in the order that these vertices were placed in $S_{t}$ to see if they produce acceptable edges. We then add in those vertices $x \notin W$ which arise from $(x, w)$ with $v=i n_{4}(w) \in S_{t}, w \notin W$, (to avoid conditioning problems).

Let $Z(v)$ be the indicator random variable for $\left(v, o u t_{4}(v)\right)$ being unacceptable and let $Z_{t}=$ $\sum_{v \in S_{t}} Z(v)$. If $Z(v)=1$ then either (i) out $4(v)$ lies on $P(\Gamma)$ and is too close to an endpoint; this has probability bounded above by $2 N_{0} /\left|V_{1}\right| \leq 401 / \log N$, or (ii) the corresponding vertex $x$ is in $W$; this has probability bounded above by $N^{3 / 4} /\left|V_{1}\right| \leq 2 N^{-1 / 4}$, or (iii) out $_{4}(v)$ lies on a small cycle. Now in a random permutation the expected number of vertices on cycles of length at most $N_{0}$ is precisely $N_{0}$ ([12]). Thus, by the Markov inequality, w.h.p. $\Gamma_{0}$ contains at most $N_{1} \log \log N_{1} /\left(2 \log N_{1}\right)+$ $N_{2} \log \log N_{2} /\left(2 \log N_{2}\right)$ vertices on small cycles. Condition on this event. Then $\mathbb{P}(Z(v)=1) \leq$ $2 \log \log N / \log N$ regardless of the history of the process and so $Z_{t}$ is stochastically dominated by $B\left(\left|S_{t}\right|, 2 \log \log N / \log N\right)$. 
Next let $X(v)$ denote the number of vertices $w$ in $V^{*} \backslash W$ such that $i_{4}(w)=v, x \notin W$ where $(v, w)$ is acceptable and $(x, w) \in \Gamma$ (if there is no such $x$ then the iteration can end early.) Let $X_{t}=\sum_{v \in S_{t}} X(v)$. Now assuming $|W| \leq N^{3 / 4}$ we see that there are $N^{\prime}=N_{1}-O(N \log \log N / \log N)$ vertices $w$ which would produce an acceptable edge provided $v=i n_{4}(w) \in S_{t}^{1}$. For these vertices, $i n_{4}(w)$ is a random choice from a set which contains $S_{t}^{1}$ and so $X_{t}$ stochastically dominates $B\left(N^{\prime},\left|S_{t}^{1}\right| / N\right)$.

Summing $1-Z(v)+X(v)$ over $v \in S_{t}$ might seem to overestimate $\left|S_{t+1}\right|$. In principle, we should subtract off the number $Y_{t}$ of vertices of $S_{t+1}$ that are counted more than once in this sum. But these arise in two ways. First, there are the pairs $v_{1}, v_{2} \in S_{t}$ with out $t_{4}\left(v_{1}\right)=$ out $_{4}\left(v_{2}\right)$. Suppose we examine $v_{1}$ before $v_{2}$. Then when we examine $v_{2}$ we find that $\operatorname{out}_{4}\left(v_{2}\right) \in W$ and so we do not get a contribution to $S_{t+1}$. Second, there is the possibility of their being $v_{1}, v_{2} \in S_{t}$ and $w$ such that $w=$ out $_{4}\left(v_{1}\right)$ and $v_{2}=i n_{4}(w)$. But in this case $w$ will only be counted once as $w \in W$ when it is time for $i n_{4}(w)$ to be examined. We can then write

$$
\left|S_{t+1}\right|=\left|S_{t}\right|-Z_{t}+X_{t}
$$

Now let $t_{0}=\lceil 1000 \log \log N\rceil, t_{1}=10 t_{0}, t_{2}=\left\lceil\log _{1.9} v+1000 \log \log N\right\rceil$,

$s_{0}=\lceil 1000 \log \log N\rceil$ and $s_{1}=\lceil 1000 \log N\rceil$.

(a) $\mathbb{P}\left(\exists t \leq t_{0}:\left|S_{t}\right| \leq s_{0}\right.$ and $\left.Z_{t}>0\right)=O\left((\log \log N)^{3} / \log N\right)$.

(b) $\quad \mathbb{P}\left(\left|\cup_{t \leq t_{0}} S_{t}^{1}\right|<0.99\left|\cup_{t \leq t_{0}} S_{t}\right||| S_{t} \mid \leq s_{0}\right.$ for $\left.t \leq t_{0}\right)=O\left((\log \log N)^{3} / \log N\right)$.

(c) $\quad \mathbb{P}\left(\sum_{t=1}^{t_{0}} X_{t} \leq s_{0} \mid S_{t} \neq \emptyset\right.$ and $\left|S_{t}\right| \leq s_{0}$ for $\left.t \leq t_{0}\right)=O\left((\log \log N)^{3} / \log N\right)$.

(d) $\mathbb{P}\left(\exists t \leq t_{1}:\left|S_{t+1}^{1}\right|<0.99\left|S_{t+1}\right| \mid S_{t} \geq 500 \log \log n\right)=O(1 / \log N)$.

(e) $\mathbb{P}\left(\exists t \leq t_{1}: 500 \log \log N \leq\left|S_{t}\right| \leq s_{1}\right.$ and $\left.Z_{t}>X_{t} / 100\right)=O(1 / \log N)$.

(f) $\mathbb{P}\left(\exists t \leq t_{1}: X_{t}<\left|S_{t}\right| / 2|| S_{t} \mid \geq 500 \log \log N\right)=O(1 / \log N)$.

(g) $\quad \mathbb{P}\left(\exists t \leq t_{1}:\left|S_{t}\right| \leq s_{1}\right.$ and $\left.X_{t} \geq 2 s_{1}\right)=O\left(N^{-2}\right)$.

(h) $\quad \mathbb{P}\left(\exists t_{1} \leq t \leq t_{2}:\left|S_{t+1}^{1}\right|<0.99\left|S_{t+1}\right| \mid S_{t} \geq s_{1}\right)=O\left(N^{-2}\right)$.

(i) $\mathbb{P}\left(\exists t \leq t_{2}:\left|S_{t}\right| \geq s_{1}\right.$ and $\left.\left|X_{t}-Z_{t}-\right| S_{t}|| \geq\left|S_{t}\right| / 10\right)=O\left(N^{-2}\right)$.

Explanations: We use the following standard inequalities for the tails of the binomial distribution:

$$
\begin{aligned}
\mathbb{P}(|B(n, p)-n p| \geq \epsilon n p) & \leq 2 e^{-\epsilon^{2} n p / 3}, \quad 0 \leq \epsilon \leq 1, \\
\mathbb{P}(B(n, p) \geq a n p) & \leq(e / a)^{a n p} .
\end{aligned}
$$

We let $\mathcal{E}_{x}, x \in\{a, b, \ldots, i\}$ be the low probability events described in (a)-(i) above.

(a) $\mathbb{P}\left(Z_{t}>0|| S_{t} \mid \leq 500 \log \log N\right)=O\left((\log \log N)^{2} / \log N\right)$ by the Markov inequality.

(b) Conditioned on $\mathcal{E}_{a}$ we have that $\left|\cup_{t \leq t_{0}} S_{t}\right| \geq t_{0}$ and $Z_{t}=0$ for $t \leq t_{0}$. Let $v_{1}, v_{2}, \ldots$ be the order in which the vertices in $\cup_{t \leq t_{0}} S_{t}$ are examined. At step $i$ with $w=\operatorname{out}_{4}\left(v_{i}\right)$ we updated $\Gamma^{\prime}=\Gamma \cup\left\{\left(v_{i}, w\right)\right\} \backslash\{(x, w)\}$ and added $x$ to $\cup_{t \leq t_{0}} S_{t}$. $x$ belongs to $V_{1}$ with probability $(1+o(1))\left|N_{1}\right| / N>0.999$. The rest follows from (A3).

(c) Conditioned on $\mathcal{E}_{a} \cap \mathcal{E}_{b}$ we have that $\left|\cup_{t \leq t_{0}} S_{t}^{1}\right| \geq 0.99 t_{0}$. Thus $\sum_{t=1}^{t_{0}} X_{t}$ dominates $B\left(0.99 t_{0} N^{\prime}, 1 / N\right)$.

(d) Similar to (b).

(e) Condition on $\left|S_{t}\right|=s \geq 500 \log \log N$ and $\mathcal{E}_{d}$. Then $Z_{t}>X_{t} / 100$ implies either that (i) $X_{t} \leq s / 10 \leq 0.99\left|S_{t}^{1}\right| / 10$ or (ii) $Z_{t}>10 s$. Both of these events have probability $O\left(1 /(\log N)^{3}\right)$.

(f) Immediate from (A3).

(g) Immediate from (A3) and (A4).

(h) Similar to (b).

(i) Similar to (c). 
Assume the occurrence of $\bigcap_{x} \overline{\mathcal{E}}_{x}$. Then $\overline{\mathcal{E}}_{a} \cap \overline{\mathcal{E}}_{c}$ implies that $\left|S_{t}\right|$ reaches size at least $500 \log \log N$ before $t$ reaches $t_{0}+1$. Once this happens, $\overline{\mathcal{E}}_{e} \cap \overline{\mathcal{E}}_{f}$ implies that $\left|S_{t}\right|$ then grows geometrically with $t$ up to time $t_{1}$ at a rate of at least 1.49 . Together with $\overline{\mathcal{E}}_{g}$ this proves that at some stage between 1 and $t_{1}$, $\left|S_{t}\right|$ reaches a size in the range $\left[s_{0}, 3 s_{0}\right] . \overline{\mathcal{E}}_{f}$ then implies that $\left|S_{t}\right|$ increases at a rate $\lambda \in[1.9,2.1]$ from then on. The lemma follows.

The total number of vertices added to $W$ in this way throughout the whole of Phase 2 is $O(\nu|S M A L L|)=o\left(N^{3 / 4}\right)$. (As we see later, we try this process once for $C \in S M A L L,|C| \leq 3$ and once or twice for $C \in S M A L L,|C| \geq 4$.)

Let $t^{*}$ denote the value of $t$ when we stop the growth of $T_{0}$. At this stage we have leaves $\Gamma_{i}$, for $i=1, \ldots, v$, each with a path of length at least $N_{0}$ (unless we have already successfully made a cycle). We now execute an In-Phase. This involves the construction of trees $T_{i}, i=1,2, \ldots v$. Assume that $P\left(\Gamma_{i}\right) \in \mathcal{P}\left(u_{0}, v_{i}\right)$. We start with $\Gamma_{i}$ and build $T_{i}$ in a similar way to $T_{0}$ except that here all paths generated end with $v_{i}$. This is done as follows: if a current NPD $\Gamma$ has $P(\Gamma) \in \mathcal{P}\left(u, v_{i}\right)$ then we consider adding an edge $(w, u) \in D_{4}$ and deleting an edge $(w, x) \in \Gamma$. Thus our trees are grown by considering edges directed into the start vertex of each $P(\Gamma)$ rather than directed out of the end vertex. Some technical changes are necessary however.

We consider the construction of our $v$ trees in two stages. First of all we grow the trees only enforcing condition $\mathrm{C}$ (ii) of success and thus allow the formation of small cycles and paths. We try to grow them to depth $t_{2}$. The growth of the $v$ trees can naturally be considered to occur simultaneously. Let $L_{i, \ell}$ denote the set of start vertices of the paths associated with the nodes at depth $\ell$ of the $i$ th tree, $i=1,2 \ldots, v, \ell=0,1, \ldots, t_{2}$. Thus $L_{i, 0}=\left\{u_{0}\right\}$ for all $i$. We prove inductively that $L_{i, \ell}=L_{1, \ell}$ for all $i, \ell$. In fact if $L_{i, \ell}=L_{1, \ell}$ then the acceptable $D_{4}$ edges have the same set of initial vertices and since all of the deleted edges are $D_{3}$-edges (enforced by C(ii)) we have $L_{i, \ell+1}=L_{1, \ell+1}$.

The probability that we succeed in constructing trees $T_{1}, T_{2}, \ldots, T_{\nu}$ is, by the analysis of Lemma 3 , $1-O\left((\log \log N)^{3} / \log N\right)$. Note that the number of nodes in each tree is $O\left(2.1^{t_{2}+1}\right)=O\left(N^{.74 \cdots}\right)$.

We now consider the fact that in some of the trees some of the leaves may have been constructed in violation of $\mathrm{C}$ (i). We imagine that we prune the trees $T_{1}, T_{2}, \ldots, T_{v}$ by disallowing any node that was constructed in violation of $\mathrm{C}(\mathrm{i})$. Let a tree be BAD if after pruning it has less than $v$ leaves and GOOD otherwise. Now an individual pruned tree has been constructed in the same manner as the tree $T_{0}$ obtained in the Out-Phase. (We have chosen $t_{2}$ to obtain $v$ leaves even at the slowest growth rate of 1.9 per node.) Thus

$$
\begin{gathered}
\mathbb{P}\left(T_{1} \text { is BAD }\right)=O\left(\frac{(\log \log N)^{3}}{\log N}\right), \\
\mathbb{E}(\text { number of BAD trees })=O\left(\frac{v(\log \log N)^{3}}{\log N}\right),
\end{gathered}
$$

and

$$
\mathbb{P}(\exists \geq v / 2 \text { BAD trees })=O\left(\frac{(\log \log N)^{3}}{\log N}\right) .
$$

Thus

$\mathbb{P}(\exists<v / 2$ GOOD trees after pruning $)$

$\leq \mathbb{P}$ (failure to construct $\left.T_{1}, T_{2}, \ldots, T_{v}\right)+\mathbb{P}(\exists \geq v / 2$ BAD trees)

$$
=O\left(\frac{(\log \log N)^{3}}{\log N}\right) \text {. }
$$


Thus with probability $1-O\left((\log \log N)^{3} / \log N\right)$ we end up with $v / 2$ sets of $v$ paths, each of length at least $100 n / \log N$ where the $i$ th set of paths all terminate in $v_{i}$. From these paths keep only those whose other endpoint $u$ lies in $V_{1}$. Then, similarly to the proof of property (h) in Lemma A.1, w.h.p. from each set we keep at least $0.99 v$ paths. The $i_{4}\left(v_{i}\right)$ are still unconditioned and hence

$$
\mathbb{P}\left(\text { no } D_{4} \text { edge closes one of these paths }\right) \leq\left(1-\frac{0.99 v}{n}\right)^{v / 2}=O\left(N^{-1 / 2}\right)
$$

Consequently, the probability that we fail to eliminate a particular small cycle $C$ after breaking an edge is $O\left((\log \log N)^{3} / \log N\right)$. If $|C| \geq 4$ then we try once or twice using independent edges of $C$ and so the probability we fail to eliminate a given small cycle $C$ is certainly $O\left(\left((\log \log N)^{3} / \log N\right)^{2}\right)$ for $|C| \geq 4$ (remember that we calculated all probabilities conditional on previous outcomes and assuming $\left.|W| \leq N^{3 / 4}\right)$.

Now the number of cycles of length 1,2 , or 3 in $D_{3}$ is asymptotically Poisson with mean $\mathrm{O}(1)$ and so there are fewer than $\log \log N$ w.h.p. Hence, since whp $|C|=O(\log N)$,

Lemma A.2. The probability that Phase 2 fails to produce a permutation digraph with minimal cycle length at least $N_{0}$ is o(1).

At this stage, we have shown that $D^{*}$ almost always contains a permutation digraph $\Pi_{2}$ in which the minimum cycle length is at least $N_{0}$. We shall refer to $\Pi_{2}$ as the Phase 2 permutation digraph.

\section{A.3 | Reassembly}

Let $D_{5}$ be the 1-in,1-out digraph left unused by the construction in the previous two sections. We will use the edges of $D_{5}$ to break-up and reassemble the cycles of $\Pi_{2}$ into a Hamilton cycle. Let $C_{1}, C_{2}, \ldots, C_{k}$ be the cycles of $\Pi_{2}$, and let $c_{i}=\left|C_{i} \cap V_{1}\right|, c_{1} \leq c_{2} \leq \cdots \leq c_{k}$. Note that $\vec{X}^{*}$ is an independent set of $D^{*}$ and so at least half the vertices of each $C_{i}$ are in $V_{1}$. If $k=1$ we can skip this phase, otherwise let $a=\frac{N}{\log N}$. For each $C_{i}$, we consider selecting a set of $m_{i}=2\left\lfloor\frac{c_{i}}{a}\right\rfloor+1$ vertices $v \in C_{i} \cap V_{1}$, and deleting the edge $(v, u)$ in $\Pi^{*}$. Let $m=\sum_{i=1}^{k} m_{i}$ and relabel (temporarily) the broken edges as $\left(v_{i}, u_{i}\right), i \in[m]$ as follows: in cycle $C_{i}$ identify the lowest numbered vertex $x_{i}$ which loses a cycle edge directed out of it. Put $v_{1}=x_{1}$ and then go round $C_{1}$ defining $v_{2}, v_{3}, \ldots, v_{m_{1}}$ in order. Then let $v_{m_{1}+1}=x_{2}$ and so on. We thus have $m$ path sections $P_{j} \in \mathcal{P}\left(u_{\phi(j)}, v_{j}\right)$ in $\Pi_{2}$ for some permutation $\phi$. We see that $\phi$ is an even permutation as all the cycles of $\phi$ are of odd length.

It is our intention to rejoin these path sections of $\Pi_{2}$ to make a Hamilton cycle using $D_{b}$, if we can. Suppose we can. This defines a permutation $\rho$ where $\rho(i)=j$ if $P_{i}$ is joined to $P_{j}$ by $\left(v_{i}, u_{\phi(j)}\right)$, where $\rho \in H_{m}$ the set of cyclic permutations on $[m]$. We will use the second moment method to show that a suitable $\rho$ exists w.h.p. A technical problem forces a restriction on our choices for $\rho$. This will produce a variance reduction in a second moment calculation.

Given $\rho$ define $\lambda=\phi \rho$. In our analysis we will restrict our attention to $\rho \in R_{\phi}=\left\{\rho \in H_{m}\right.$ : $\left.\phi \rho \in H_{m}\right\}$. If $\rho \in R_{\phi}$ then we have not only constructed a Hamilton cycle in $\Pi_{2} \cup D_{5}$, but also in the auxiliary digraph $\Lambda$, whose edges are $(i, \lambda(i))$.

Lemma A.3. $\quad(m-2) ! \leq\left|R_{\phi}\right| \leq(m-1) !$

Proof. We grow a path $1, \lambda(1), \lambda^{2}(1), \ldots, \lambda^{r}(1) \ldots$ in $\Lambda$, maintaining feasibility in the way we join the path sections of $\Pi_{2}$ at the same time. 
We note that the edge $(i, \lambda(i))$ of $\Lambda$ corresponds in $D_{5}$ to the edge $\left(v_{i}, u_{\phi \rho(i)}\right)$. In choosing $\lambda(1)$, we must avoid not only 1 but also $\phi(1)$ since $\lambda(1)=1$ implies $\rho(1)=1$. Thus there are $m-2$ choices for $\lambda(1)$ since $\phi(1) \neq 1$ from the definition of $m_{1}$.

In general, having chosen $\lambda(1), \lambda^{2}(1), \ldots, \lambda^{r}(1), 1 \leq r \leq m-3$ our choice for $\lambda^{r+1}(1)$ is restricted to be different from these choices and also 1 and $\ell$ where $u_{\ell}$ is the initial vertex of the path terminating at $v_{\lambda^{r}(1)}$ made by joining path sections of $\Pi_{2}$. Thus there are either $m-(r+1)$ or $m-(r+2)$ choices for $\lambda^{r+1}(1)$ depending on whether or not $\ell=1$.

Hence, when $r=m-3$, there may be only one choice for $\lambda^{m-2}(1)$, the vertex $h$ say. After adding this edge, let the remaining isolated vertex of $\Lambda$ be $w$. We now need to show that we can complete $\lambda$, $\rho$ so that $\lambda, \rho \in H_{m}$.

Which vertices are missing edges in $\Lambda$ at this stage? Vertices $1, w$ are missing in-edges, and $h, w$ out-edges. Hence, the path sections of $\Pi_{2}$ are joined so that either

$$
u_{1} \rightarrow v_{h}, \quad u_{w} \rightarrow v_{w} \quad \text { or } \quad u_{1} \rightarrow v_{w}, \quad u_{w} \rightarrow v_{h}
$$

The first case can be (uniquely) feasibly completed in both $\Lambda$ and $\Pi_{2}$ by setting $\lambda(h)=w, \lambda(w)=1$. Completing the second case to a cycle in $\Pi_{2}$ means that

$$
\lambda=\left(1, \lambda(1), \ldots, \lambda^{m-2}(1)\right)(w)
$$

and thus $\lambda \notin H_{m}$. We show this case cannot arise.

$\lambda=\phi \rho$ and $\phi$ is even implies that $\lambda$ and $\rho$ have the same parity. On the other hand, $\rho \in H_{m}$ has a different parity to $\lambda$ in (A5) which is a contradiction.

Thus there is a (unique) completion of the path in $\Lambda$.

Let $H$ stand for the union of the permutation digraph $\Pi_{2}$ and $D_{5}$. We finish our proof by proving

Lemma A.4. $\mathbb{P}(H$ does not contain a Hamilton cycle $)=o(1)$.

Proof. Let $X$ be the number of Hamilton cycles in $H$ obtainable by deleting edges as above, rearranging the path sections generated by $\phi$ according to those $\rho \in R_{\phi}$ and if possible reconnecting all the sections using edges of $D_{5}$. We will use the inequality

$$
\mathbb{P}(X>0) \geq \frac{\mathbb{E}(X)^{2}}{\mathbb{E}\left(X^{2}\right)}
$$

Probabilities in (A6) are thus with respect to the space of $D_{5}$ choices.

Now the definition of the $m_{i}$ yields that

$$
\frac{2 N}{a}-k \leq m \leq \frac{2 N}{a}+k
$$

and so

$$
\text { (1.99) } \log N \leq m \leq(2.01) \log N \text {. }
$$

Also

$$
k \leq \frac{\log N}{200}, m_{i} \geq 199, \text { and } \frac{c_{i}}{m_{i}} \geq \frac{a}{2.01}, \quad 1 \leq i \leq k
$$


Let $\Omega$ denote the set of possible cycle rearrangements. $\omega \in \Omega$ is a success if $D_{5}$ contains the edges needed for the associated Hamilton cycle. Let $b_{i}$ be the number of deleted edges $\left(v_{i}, u_{i}\right)$ with $u_{i} \notin V_{1}$ and $b=\sum_{i=1}^{k} b_{i}$. Observe that if $u_{i} \in V_{1}$ then $\left(v_{i}, u_{i}\right) \in E\left(D_{5}\right) \backslash E\left(D_{4}\right)$ with probability $1-\left(1-\frac{1}{N_{1}}\right)^{2}$ while if $u_{j} \notin V_{1}$ then $\left(v_{i}, u_{j}\right) \in E\left(D_{5}\right) \backslash E\left(D_{4}\right)$ with probability $\frac{1}{N_{1}}$.

For a fixed $\alpha>0$, we have

$$
n e^{-c / 2} \geq N-N_{1} \geq b \geq \sum_{j: b_{j} \geq \alpha\left|C_{j}\right|} b_{j} \geq \alpha \sum_{j: b_{j} \geq \alpha\left|C_{j}\right|}\left|C_{j}\right| .
$$

Putting $\alpha=10^{-3}$ we see that at most $1000 n e^{-c / 2} \leq e^{-c / 3} N$ vertices lie on a cycle $C_{i}$ with more than $0.001\left|C_{i}\right|$ vertices that do not lie in $V_{1}$. Therefore $b$ is stochastically dominated by $(1+o(1))\left(e^{-c / 3} m+\right.$ $\operatorname{Bin}\left(\left(1-e^{-c / 3}\right) m, 10^{-3}\right)$. Hence $\mathbb{P}(b>0.01 m)=o(1)$. Thus,

$$
\begin{aligned}
& \mathbb{E}(X)=\sum_{\omega \in \Omega} \mathbb{P}(\omega \text { is a success }) \\
& =\sum_{\omega \in \Omega}\left(1-\left(1-\frac{1}{N_{1}}\right)^{2}\right)^{m-b(\omega)}\left(\frac{1}{N_{1}}\right)^{b(\omega)} \\
& \geq(1-o(1))\left(\frac{2}{N_{1}}\right)^{m} 2^{-0.01 m} \cdot \mathbb{P}(b \leq 0.01 m)(m-2) ! \prod_{i=1}^{k}\left(\begin{array}{c}
c_{i} \\
m_{i}
\end{array}\right) \\
& \geq \frac{1-o(1)}{m \sqrt{m}}\left(\frac{2 m}{e N_{1}}\right)^{m} \prod_{i=1}^{k}\left(\left(\frac{c_{i} e^{1-1 / 12 m_{i}}}{m_{i}^{1+\left(1 / 2 m_{i}\right)}}\right)^{m_{i}}\left(\frac{1-2 m_{i}^{2} / c_{i}}{\sqrt{2 \pi}}\right)\right) 2^{-0.01 m} \\
& \geq \frac{(1-o(1))(2 \pi)^{-m / 398} e^{-k / 12}}{m \sqrt{m}}\left(\frac{2 m}{e N_{1}}\right)^{m} \prod_{i=1}^{k}\left(\frac{c_{i} e}{(1.02) m_{i}}\right)^{m_{i}} 2^{-0.01 m} \\
& \geq \frac{(1-o(1))(2 \pi)^{-m / 398}}{n^{1 / 1200} m \sqrt{m}}\left(\frac{2 m}{e N_{1}}\right)^{m}\left(\frac{e a}{2.01 \times 1.02}\right)^{m} 2^{-0.01 m} \\
& \geq \frac{(1-o(1))(2 \pi)^{-m / 398}}{N_{1}^{1 / 1200} m \sqrt{m}}\left(\frac{3.98}{2.0502}\right)^{m} 2^{-0.01 m} \\
& \geq N_{1}^{1.3} \text {. }
\end{aligned}
$$

Let $A, A^{\prime}$ be two sets of selected edges which have been deleted in $\Pi_{2}$ and whose path sections have been rearranged into Hamilton cycles according to $\rho, \rho^{\prime}$, respectively. Let $B, B^{\prime}$ be the corresponding sets of edges which have been added to make the Hamilton cycles. What is the interaction between these two Hamilton cycles?

Let $s=\left|A \cap A^{\prime}\right|$ and $t=\left|B \cap B^{\prime}\right|$. Now $t \leq s$ since if $(v, u) \in B \cap B^{\prime}$ then there must be a unique $(\tilde{v}, u) \in A \cap A^{\prime}$ which is the unique $\Pi_{2}$-edge into $u$. We claim that $t=s$ implies $t=s=m$ and $(A, \rho)=\left(A^{\prime}, \rho^{\prime}\right)$. (This is why we have restricted our attention to $\rho \in R_{\phi}$.) Suppose then that $t=s$ and $\left(v_{i}, u_{i}\right) \in A \cap A^{\prime}$. Now the edge $\left(v_{i}, u_{\lambda(i)}\right) \in B$ and since $t=s$ this edge must also be in $B^{\prime}$. But this implies that $\left(v_{\lambda(i)}, u_{\lambda(i)}\right) \in A^{\prime}$ and hence in $A \cap A^{\prime}$. Repeating the argument we see that $\left(v_{\lambda^{k}(i)}, u_{\lambda^{k}(i)}\right) \in A \cap A^{\prime}$ for all $k \geq 0$. But $\lambda$ is cyclic and so our claim follows.

We adopt the following notation. Let $\left\langle s, t>\right.$ denote $\left|A \cap A^{\prime}\right|=s$ and $\left|B \cap B^{\prime}\right|=t$. So

$$
\mathbb{E}\left(X^{2}\right) \leq \mathbb{E}(X)+(1+o(1)) \sum_{A \in \Omega}\left(\frac{2}{N_{1}}\right)^{m} \sum_{\substack{A^{\prime} \in \Omega \\ B^{\prime} \cap B=\emptyset}}\left(\frac{2}{N_{1}}\right)^{m}
$$




$$
\begin{aligned}
& +(1+o(1)) \sum_{A \in \Omega}\left(\frac{2}{N_{1}}\right)^{m} \sum_{s=2}^{m} \sum_{t=1}^{m-1} \sum_{\substack{A^{\prime} \in \Omega \\
<s, t>}}\left(\frac{2}{N_{1}}\right)^{m-t} \\
= & \mathbb{E}(X)+E_{1}+E_{2} \text { say. }
\end{aligned}
$$

Clearly

$$
E_{1} \leq(1+o(1)) \mathbb{E}(X)^{2}
$$

For given $\rho$, how many $\rho^{\prime}$ satisfy the condition $\left\langle s, t>\right.$ ? Previously $\left|R_{\phi}\right| \geq(m-2)$ ! and now given $<s, t>,\left|R_{\phi}(s, t)\right| \leq(m-t-1)$ !, (consider fixing $t$ edges of $\left.\Lambda^{\prime}\right)$.

Thus

$$
E_{2} \leq \mathbb{E}(X)^{2} \sum_{s=2}^{m} \sum_{t=1}^{s-1}\left(\begin{array}{c}
s \\
t
\end{array}\right)\left[\sum_{\sigma_{1}+\ldots+\sigma_{k}=s} \prod_{i=1}^{k} \frac{\left(\begin{array}{c}
m_{i} \\
\sigma_{i}
\end{array}\right)\left(\begin{array}{c}
c_{i}-m_{i} \\
m_{i}-\sigma_{i}
\end{array}\right)}{\left(\begin{array}{c}
c_{i} \\
m_{i}
\end{array}\right)}\right] \frac{(m-t-1) !}{(m-2) !}\left(\frac{N_{1}}{2}\right)^{t}
$$

For the above expression observe that given $A \cap A^{\prime}$ there are $\left(\begin{array}{l}s \\ t\end{array}\right)$ choices for $B \cap B^{\prime}$. Thereafter given $A$ and $\sigma_{i}$ there are $\left(\begin{array}{c}m_{i} \\ \sigma_{i}\end{array}\right)$ ways to choose $A \cap A^{\prime} \cap C_{i}$ and $\left(\begin{array}{c}c_{i}-m_{i} \\ m_{i}-\sigma_{i}\end{array}\right)$ ways to choose the rest of $B_{i}^{\prime} \cap C_{i}$.

Now

$$
\begin{aligned}
\frac{\left(\begin{array}{c}
c_{i}-m_{i} \\
m_{i}-\sigma_{i}
\end{array}\right)}{\left(\begin{array}{c}
c_{i} \\
m_{i}
\end{array}\right)} & \leq \frac{\left(\begin{array}{c}
c_{i} \\
m_{i}-\sigma_{i}
\end{array}\right)}{\left(\begin{array}{c}
c_{i} \\
m_{i}
\end{array}\right)} \\
& \leq(1+o(1))\left(\frac{m_{i}}{c_{i}}\right)^{\sigma_{i}} \exp \left\{-\frac{\sigma_{i}\left(\sigma_{i}-1\right)}{2 m_{i}}\right\} \\
& \leq(1+o(1))\left(\frac{2.01}{a}\right)^{\sigma_{i}} \exp \left\{-\frac{\sigma_{i}\left(\sigma_{i}-1\right)}{2 m_{i}}\right\},
\end{aligned}
$$

where the $o(1)$ term is $O\left((\log N)^{3} / N\right)$. Also

$$
\begin{gathered}
\sum_{i=1}^{k} \frac{\sigma_{i}^{2}}{2 m_{i}} \geq \frac{s^{2}}{2 m} \quad \text { for } \sigma_{1}+\cdots+\sigma_{k}=s \\
\sum_{i=1}^{k} \frac{\sigma_{i}}{2 m_{i}} \leq \frac{k}{2}
\end{gathered}
$$

and

$$
\sum_{\sigma_{1}+\ldots+\sigma_{k}=s} \prod_{i=1}^{k}\left(\begin{array}{c}
m_{i} \\
\sigma_{i}
\end{array}\right)=\left(\begin{array}{c}
m \\
s
\end{array}\right)
$$

Hence

$$
\frac{E_{2}}{\mathbb{E}(X)^{2}} \leq(1+o(1)) e^{k / 2} \sum_{s=2}^{m} \sum_{t=1}^{s-1}\left(\begin{array}{c}
s \\
t
\end{array}\right) \exp \left\{-\frac{s^{2}}{2 m}\right\}\left(\frac{2.01}{a}\right)^{s}\left(\begin{array}{c}
m \\
s
\end{array}\right) \frac{(m-t-1) !}{(m-2) !}\left(\frac{N_{1}}{2}\right)^{t}
$$




$$
\begin{aligned}
& \leq(1+o(1)) N^{.005} \sum_{s=2}^{m} \sum_{t=1}^{s-1}\left(\begin{array}{l}
s \\
t
\end{array}\right) \exp \left\{-\frac{s^{2}}{2 m}\right\}\left(\frac{2.01}{a}\right)^{s} \frac{m^{s-(t-1)}}{(s-1) !}\left(\frac{N_{1}}{2}\right)^{t} \\
& =(1+o(1)) N^{.005} \sum_{s=2}^{m}\left(\frac{2.01}{a}\right)^{s} \frac{m^{s}}{s !} \exp \left\{-\frac{s^{2}}{2 m}\right\} m \sum_{t=1}^{s-1}\left(\begin{array}{l}
s \\
t
\end{array}\right)\left(\frac{N_{1}}{2 m}\right)^{t} \\
& \leq(1+o(1))\left(\frac{2 m^{3}}{N^{3} 99}\right) \sum_{s=2}^{m}\left(\frac{(2.01) N_{1} \exp \{-s / 2 m\}}{2 a}\right)^{s} \frac{1}{s !} \\
& =o(1) .
\end{aligned}
$$

To verify that the RHS of (A10) is $o(1)$, we can split the summation into

$$
S_{1}=\sum_{s=2}^{\lfloor m / 4\rfloor}\left(\frac{(2.01) N_{1} \exp \{-s / 2 m\}}{2 a}\right)^{s} \frac{1}{s !}
$$

and

$$
S_{2}=\sum_{s=\lfloor m / 4\rfloor+1}^{m}\left(\frac{(2.01) N_{1} \exp \{-s / 2 m\}}{2 a}\right)^{s} \frac{1}{s !} .
$$

Ignoring the term $\exp \{-s / 2 m\}$ we see that

$$
S_{1} \leq \sum_{s=2}^{\lfloor(.5025) \log N\rfloor} \frac{((1.005) \log N)^{s}}{s !}=o\left(N^{9 / 10}\right)
$$

since this latter sum is dominated by its last term.

Finally, using $\exp \{-s / 2 m\}<e^{-1 / 8}$ for $s>m / 4$ we see that

$$
S_{2} \leq N^{(1+o(1)) 1.005) e^{-1 / 8}}<N^{9 / 10} .
$$

The result follows from (A6) to (A10). 